Un ACERCAMIENTO AL CAMPO ARGENTINO DE PRODUCCIÓN DE CONOCIMIENTO EDUCATIVO A TRAVÉS DE LAS PUBLICACIONES EN

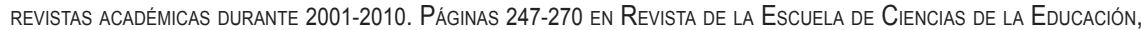
AÑO 10, NÚMERO 9, ENERO A DICIEMBRE DE 2014. ISSN 1851-6297. ISSN EN LINEA 2362-3349.

\title{
UN ACERCAMIENTO AL CAMPO ARGENTINO DE PRODUCCIÓN DE CONOCIMIENTO EDUCATIVO A TRAVÉS DE LAS PUBLICACIONES EN REVISTAS ACADÉMICAS DURANTE 2001-2010
}

\author{
Por Jorge M. Gorostiaga (Universidad Nacional de San Martín - CONICET)*, \\ Argentina. \\ jorgegoros@gmail.com \\ Gimena Nieto (Universidad Nacional de San Martín)**, Argentina. \\ gimenanieto@yahoo.com.ar \\ Florencia Cueli (Universidad Nacional de San Martín) ${ }^{* * *}$, Argentina. \\ florenciacueli@gmail.com
}

Recibido: 23/06/2014 Aceptado: 25/08/2014

\section{Resumen}

El objetivo del artículo es contribuir al estudio del campo de la producción de conocimiento educativo en la Argentina. Para ello, se analizan los artículos sobre educación publicados por investigadores pertenecientes a instituciones argentinas durante el período 2001-2010 en un conjunto de revistas académicas -incluyendo las revistas especializadas en educación, una selección de revistas de ciencias sociales y humanas, y una selección de revistas extranjeras- en lo referido a la productividad por regiones geográficas, sectores institucionales y áreas/temáticas de especialización.

El aumento del volumen global de artículos a lo largo de la década indicaría cierta consolidación del campo, aunque persisten algunos desbalances significativos en cuanto a la distribución regional, institucional y disciplinaria de los artículos. Como

* Doctor en Análisis Social y Comparado de la Educación (Universidad de Pittsburgh). Profesor de Grado y Postgrado en la Universidad Nacional de San Martín. Profesor de Postgrado en la Universidad Torcuato Di Tella. Investigador Adjunto del Consejo Nacional de Investigaciones Científicas y Técnicas (CONICET).

** Licenciada en Educación y Profesora en Ciencias de la Educación (Universidad Nacional de San Martín). Profesora de Grado en la Licenciatura en Educación Escuela de Humanidades, Universidad Nacional de San Martín.

${ }^{* * *}$ Licenciada en Educación (Universidad Nacional de San Martín). Becaria del Consejo Interuniversitario Nacional. 
Revista de la Escuela de Ciencias de la Educación, año 10, número 9, enero a diciembre de 2014. Páginas 247-270. ISSN 1851-6297. ISSN EN LINEA 2362-3349. UN ACERCAMIENTO AL CAMPO ARGENTINO DE PRODUCCIÓN DE CONOCIMIENTO

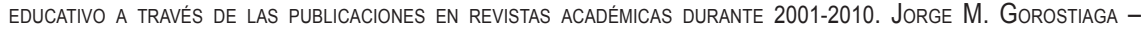
Gimena Nieto - Florencia Cueli.

rasgos más salientes, se registra un claro predominio de las regiones Metropolitana y Centro-Oeste y del sector de universidades públicas, dentro del cual se destaca la hegemonía de la Universidad de Buenos Aires. Por otra parte, en términos de temáticas y áreas disciplinarias, es notable la baja participación de Educación de Adultos y de Economía de la Educación.

\section{Palabras Clave}

Producción de conocimiento - campo académico de la educación - revistas académicas - Argentina.

\section{Abstract}

The objective of this article is to contribute to the study of the field of educational knowledge production in Argentina. To this end, we analyze the articles on education published by researchers from Argentine institutions between 2001 and 2010 in a group of journals -including journals specialized in education, a selection of social sciences and humanities journals, and a selection of foreign journals-with regard to productivity along geographic regions, institutional sectors and topics/areas of specialization.

The growth of the global bulk of articles during the decade may indicate a consolidation of the field, even though some significant imbalances in terms of regional, institutional and disciplinary distribution of articles still persist. The most salient features are: the manifest prevalence of Metropolitan and Center-West regions, and of the public universities sector, in which the hegemony of University of Buenos Aires stands out. With regard to topics and disciplines, it is noteworthy the low participation of Adult Education and Economics of Education.

\section{Key Words}

Knowledge production - Academic field of education - Academic journals Argentina.

\section{Introducción}

En este artículo analizamos la producción de conocimiento sobre educación de investigadores pertenecientes a instituciones argentinas durante el período 2001-2010 en un conjunto de revistas académicas, incluyendo las revistas especializadas en educación, una selección de revistas de ciencias sociales y humanas, y una selección de revistas extranjeras. Las revistas académicas son un elemento central en la conformación y en la dinámica de las disciplinas científicas tanto a nivel mundial como al interior de cada país, ya que constituyen el medio privilegiado de comunicación de los resultados de investigación y de los desarrollos disciplinares (Schriewer \& Keiner, 1997; Waldegg, 1997), aunque debe tenerse en cuenta que una parte importante de la producción de conocimiento es canalizada por otros medios de difusión como libros, revistas de divulgación, informes, ponencias en congresos, tesis de grado y de posgrado, 
Revista de la Escuela de Ciencias de la Educación, año 10, número 9, enero a diciembre de 2014. Páginas 247-270. ISSN 1851-6297. ISSN EN LINEA 2362-3349. UN ACERCAMIENTO AL CAMPO ARGENTINO DE PRODUCCIÓN DE CONOCIMIENTO

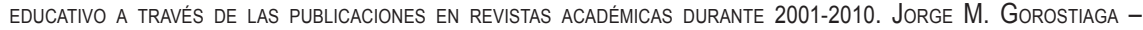
Gimena Nieto - Florencia Cueli.

y documentos de trabajo. Nuestro trabajo utiliza algunos criterios bibliométricos que posibilitan "el acercamiento a la situación de un campo científico" (Tarres, $2009, s / n)$, teniendo en cuenta antecedentes sobre este tipo de aproximaciones específicamente para la educación (Alvarenga, 2000; Galán \& Rojas, 1995; Merodo, Atairo, Stagno \& Palamidessi, 2007). El objetivo del artículo es contribuir al estudio del campo de la producción de conocimiento educativo en la Argentina (1), particularmente en lo referido a la productividad por regiones geográficas, sectores institucionales y áreas/temáticas de especialización.

Desde el retorno a la democracia, el campo argentino de producción de conocimientos especializados en educación ha crecido en términos cuantitativos (tanto en cantidad de actores como en generación de proyectos de investigación y publicaciones) y se ha diversificado en términos institucionales, aunque con marcadas insuficiencias en su base profesional y en la diferenciación e institucionalización de especialidades y disciplinas (Palamidessi, Galarza \& Cardini, 2012), insuficiencias que en algunos aspectos se vinculan, y en otros se suman, a la debilidad general de la profesión académica en la Argentina (Marquina, 2012). En términos comparados, es un campo menos institucionalizado y estructurado que el de Brasil o México, tanto en su dinámica interna como en sus relaciones con la esfera estatal (Palamidessi, Gorostiaga \& Suasnábar, 2014).

Durante la década de 2000 , se mantuvieron algunas tendencias del decenio anterior: la consolidación de un núcleo estable de docentes universitarios y de mecanismos de incentivos y evaluación del trabajo académico (Marquina, 2012), la expansión de las carreras de posgrado en diversas áreas del conocimiento -incluyendo educación (De la Fare, 2008) - y la creación de nuevas universidades, aunque ahora preponderantemente en el sector estatal. Además, se implementaron políticas que favorecieron el desarrollo de la profesión académica y, concomitantemente, de la investigación educativa. Así, el sector de las universidades públicas -tradicionalmente el principal productor de conocimiento educativo en el país- obtuvo una recomposición presupuestaria y salarial. A su vez, se registró un significativo crecimiento del sistema de ciencia y tecnología, con el aumento de subsidios para actividades de investigación y desarrollo tecnológico, la ampliación del número de investigadores y becarios en el Consejo Nacional de Investigaciones Científicas y Técnicas (CONICET), la repatriación de investigadores, y la creación del Ministerio de Ciencia y Tecnología.

En las siguientes secciones presentamos las fuentes y procedimientos empleados en nuestro estudio, seguidos por los resultados del análisis de los artículos en cuanto a volumen global y a su distribución por regiones geográficas, sectores institucionales y áreas disciplinarias, replicando en gran medida el estudio de Merodo et al. (2007), el cual investigó la producción de libros y artículos de revistas especializadas para el período 1997-2003. Vale aclarar que el análisis no aborda la cuestión de la calidad de los artículos publicados; tampoco se limita a los trabajos que son productos de investigación en sentido 
Revista de la Escuela de Ciencias de la Educación, año 10, número 9, enero a diciembre de 2014. Páginas 247-270. ISSN 1851-6297. ISSN EN LINEA 2362-3349. UN ACERCAMIENTO AL CAMPO ARGENTINO DE PRODUCCIÓN DE CONOCIMIENTO

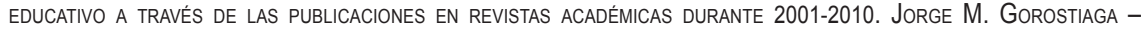
Gimena Nieto - Florencia Cueli.

estricto, sino que apunta a la producción de conocimiento educativo en forma amplia, incluyendo artículos de tipo ensayístico. A partir de los datos presentados, las conclusiones señalan algunas tendencias generales sobre los patrones de producción académica así como continuidades y cambios respecto al estudio de Merodo et al. (2007).

\section{Fuentes y Procedimientos}

El estudio se basa en tres fuentes de datos: a) revistas académicas argentinas especializadas en educación; b) revistas argentinas de ciencias sociales y humanas no especializadas en educación; y c) revistas académicas extranjeras especializadas en educación. Para cada una de las fuentes se construyó una base de datos de los artículos en los que hubiera al menos un autor de pertenencia a una institución argentina, abarcando el período 2001-2010 (2).

En el caso de revistas argentinas sobre educación, la intención fue cubrir todo el universo de revistas académicas especializadas, identificándose un total de 30 (3). Por revistas académicas entendemos aquellas que declaran poseer mecanismos de arbitraje o selección en base a criterios de calidad para la aceptación de artículos (4) y que son editadas por universidades o asociaciones científicas. Los números analizados fueron obtenidos en las bibliotecas Nacional de Maestros, de la Facultad de Filosofía y Letras de la Universidad de Buenos Aires (UBA) y de la Facultad de Humanidades y Ciencias de la Educación de la Universidad Nacional de La Plata (UNLP), de las páginas web de las propias revistas y de las páginas web de bases de revistas como REDALYC, SCIELO, etc. Cabe observar que en el campo argentino de la educación, las revistas científicas presentan una significativa variedad en cuanto a los formatos y lógicas de difusión que adoptan, lo cual pareciera reflejar la propia heterogeneidad del campo (Palamidessi \& Devetac, 2007). Así, algunas revistas se proponen, fundamentalmente, difundir la producción de los miembros de la institución editora de la revista (sea una universidad o una asociación científica) mientras otras se orientan a un diálogo más amplio con el campo; al mismo tiempo, unas se dedican a la educación en general y otras a recortes específicos de estudio (como la historia de la educación, la enseñanza de la física, etc.). La heterogeneidad del campo se refleja también en lo que es considerado un artículo académico: una simple mirada a las revistas permite apreciar una gran disparidad en cuanto a la extensión y estructura de los artículos publicados.

Para la selección de revistas de ciencias sociales y humanas, se realizó un relevamiento de las publicaciones de universidades públicas y privadas y de asociaciones académicas, y de la base de SCIELO Argentina. Se seleccionaron revistas que publican con cierta regularidad sobre educación o que, en el período estudiado, hubieran publicado al menos un número especial dedicado a educación, resultando un total de doce revistas (5). La heterogeneidad en 
Revista de la Escuela de Ciencias de la Educación, año 10, número 9, enero a diciembre de 2014. Páginas 247-270. ISSN 1851-6297. ISSN EN LINEA 2362-3349. UN ACERCAMIENTO AL CAMPO ARGENTINO DE PRODUCCIÓN DE CONOCIMIENTO

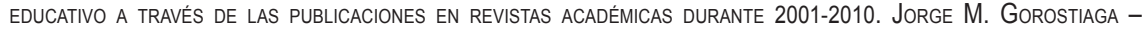
Gimena Nieto - Florencia Cuell.

los formatos y lógicas de difusión que se señaló para las revistas argentinas de educación también se verifica para esta selección o muestra de revistas, al menos en lo que respecta a los artículos sobre educación publicados por ellas.

Para las revistas extranjeras, el criterio general fue incluir revistas de alto prestigio, privilegiando aquéllas en las que hubiera antecedentes de publicaciones de autores de instituciones argentinas. Para ello, se tomó en cuenta (revisando y ampliando) la selección de revistas del estudio de Merodo et al. (2007), realizada en base a consultas a referentes académicos y a trabajos sobre revistas educativas. Se relevaron 42 revistas de Brasil, España, Estados Unidos, Gran Bretaña, México y organismos internacionales (6).

Para cada número de revista analizado se identificaron los artículos sobre educación firmados por al menos un autor con sede en una institución argentina, excluyendo las reediciones de artículos, los editoriales, las presentaciones, las reseñas y las secciones del tipo "opiniones", "artículos sin referato" o "resúmenes de tesis". De cada artículo identificado se extrajo la información de: título del artículo; nombre de autor/es; institución/es de pertenencia; palabras clave; resumen (cuando estaba disponible). Así, se construyeron las tres bases de datos de artículos de revistas: argentinas de educación; argentinas de ciencias sociales y humanas; y extranjeras de educación.Sobre cada base se aplicaron procedimientos para establecer la distribución de los artículos por institución, sector institucional y región geográfica. Para esto se consideró la institución principal (la reportada en primer lugar en la publicación) del primer autor de cada artículo. Para 24 artículos de las revistas argentinas de educación y un artículo de las revistas extranjeras no fue posible determinar la pertenencia institucional de sus autores, por lo cual no fueron tenidos en cuenta para el análisis de distribución regional y por sector institucional. La distribución regional se determinó de acuerdo con la división territorial de las Consejos de Planificación Regional de la Educación Superior (CPRES) (7). La clasificación de sectores institucionales se organizó a partir de las siguientes categorías: universidades públicas (incluye nacionales y provinciales); universidades privadas; centros independientes (incluye organismos sindicales); institutos terciarios y secundarios; organismos de ciencia y tecnología; organismos gubernamentales; y organismos internacionales. En los casos de doble pertenencia universidadCONICET (8), se optó por tomar la universidad -aun en los casos en que se reporta CONICET como institución principal- para poder realizar un análisis más preciso de la distribución institucional dentro del sector de universidades públicas y privadas.

En segundo lugar, se realizó un análisis de los títulos y, en los casos en que estaban disponibles, de los resúmenes de los artículos para determinar el área disciplinaria de cada uno. Las áreas disciplinarias utilizadas son una adaptación de las categorías definidas para el Directorio de Proyectos de Incentivos de la Secretaría de Políticas Universitarias del año 2001 (9). 
Revista de la Escuela de Ciencias de la Educación, año 10, número 9, enero a diciembre de 2014. Páginas 247-270. ISSN 1851-6297. ISSN EN LINEA 2362-3349. UN ACERCAMIENTO AL CAMPO ARGENTINO DE PRODUCCIÓN DE CONOCIMIENTO EDUCATIVO A TRAVÉS DE LAS PUBLICACIONES EN REVISTAS ACADÉMICAS DURANTE 2001-2010. JoRGe M. GoROSTIAGA Gimena Nieto - Florencia Cueli.

\section{Análisis de la producción}

Se identificó un total de 1514 artículos en revistas argentinas, 291 artículos en revistas de ciencias sociales y humanas, y 344 artículos en revistas extranjeras. Respecto a la base de artículos de revistas argentinas, la tendencia ascendente de cantidad de artículos a lo largo del decenio (véase Cuadro 1) se explica, en buena medida, por el aumento sostenido de la cantidad de revistas que se publican, pero también puede relacionarse a un contexto económico de recuperación después de la crisis de 2001-2002, dado que la supervivencia y regularidad de las revistas parece ser muy sensible al cambiante escenario económico nacional (Palamidessi y Devetac, 2007).

\section{CUADRO 1}

\section{Cantidad de artículos según tipo de revista por año}

\begin{tabular}{|c|c|c|c|}
\hline Año & $\begin{array}{c}\text { Revistas } \\
\text { de educación }\end{array}$ & $\begin{array}{c}\text { Revistas de ciencias } \\
\text { sociales y humanas }\end{array}$ & $\begin{array}{c}\text { Revistas } \\
\text { extranjeras }\end{array}$ \\
\hline 2001 & 99 & 14 & 23 \\
\hline 2002 & 123 & 13 & 23 \\
\hline 2003 & 108 & 12 & 41 \\
\hline 2004 & 131 & 55 & 40 \\
\hline 2005 & 146 & 25 & 36 \\
\hline 2006 & 185 & 31 & 30 \\
\hline 2007 & 156 & 27 & 44 \\
\hline 2008 & 206 & 43 & 37 \\
\hline 2009 & 187 & 35 & 30 \\
\hline 2010 & 173 & 36 & 40 \\
\hline Total & 1514 & 291 & 344 \\
\hline
\end{tabular}

Las cifras de artículos publicados en revistas de ciencias sociales y humanas y en revistas extranjeras, por su parte, también parecen seguir una tendencia ascendente, aunque no tan clara y lineal como la de artículos en revistas de educación argentinas. La cantidad de artículos identificados en revistas de ciencias sociales y humanas -teniendo en cuenta que se trata de una muestra muy pequeña de revistas- indica que la propensión a publicar en revistas académicas no especializadas en educación es significativa. Por su parte, el volumen de artículos en revistas extranjeras, las cuales presentan en 
Revista de la Escuela de Ciencias de la Educación, año 10, número 9, enero a diciembre de 2014. Páginas 247-270. ISSN 1851-6297. ISSN EN LINEA 2362-3349. UN ACERCAMIENTO AL CAMPO ARGENTINO DE PRODUCCIÓN DE CONOCIMIENTO

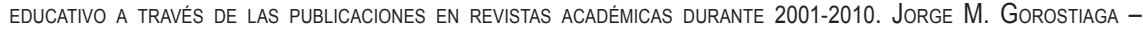
Gimena Nieto - Florencia Cueli.

general niveles más altos de exigencia académica, revela una considerable disposición y capacidad para acceder a ese ámbito de difusión científica.

Uno de los aspectos cuantitativos sobresalientes dentro de los artículos de revistas argentinas de educación es el peso de Alternativas Serie Espacio Pedagógico de la Universidad Nacional de San Luis (UNSL): el 18\% del total de los artículos identificados pertenecen a dicha revista; seguida por Cuadernos de Educación, de la Universidad Nacional de Córdoba (UNC), con casi un 9\%. El peso de ambas revistas se explica por dos factores: 1) habiendo sido creadas antes del 2001, cubren todo el período estudiado; y 2) publican una alta cantidad de artículos por número, típicamente más de 15 y en algunos casos más de 20. En el caso de Alternativas, se agrega el hecho de que es la única que publica 3 o 4 números por año.

Entre las revistas de ciencias sociales y humanas se destaca Fundamentos en Humanidades (editada por la UNSL), ya que es la que dedicó mayor parte de su producción total al área educativa $(36,27 \%)$ y la que más artículos sobre educación - 70 artículos, que representan un $24 \%$ de la base- publicó en el período. Detrás de ella, las revistas que más artículos sobre educación aportan son el Anuario de Investigaciones (UBA, Facultad de Psicología), Cuadernos de la Facultad de Humanidades y Ciencias Sociales (Universidad Nacional de Jujuy - UNJu) y Ciencia, Docencia y Tecnología (Universidad Nacional de Entre Ríos - UNER), con 45, 44 y 43 artículos, respectivamente.

Dentro de las revistas extranjeras, las de España son las que concentran mayor producción (96 artículos en 10 revistas), pero las de México tienen el promedio más alto de artículos por revista (19, correspondientes a 95 artículos en cinco revistas), sobresaliendo la Revista Mexicana de Investigación Educativa, con un promedio de un artículo de autor perteneciente a una institución argentina por número. También la Revista Iberoamericana de Educación (editada por la Organización de Estados Iberoamericanos), Enseñanza de las Ciencias (España), Revista Electrónica Iberoamericana sobre Calidad, Eficacia y Cambio en Educación (España), Avaliação (Brasil) y Archivos Analíticos de Política Educativa (EEUU) muestran una presencia muy alta de artículos provenientes de instituciones argentinas.

El Cuadro 2 presenta la distribución por región. Para los artículos de revistas argentinas, dominan las regiones Centro-Oeste y Metropolitana, concentrando en conjunto casi un $60 \%$ del total, seguidas por las regiones Bonaerense y Centro-Este, ambas con un porcentaje casi idéntico, entre el 11 y el $12 \%$. En las revistas de ciencias sociales y humanas, por su parte, hay un claro predominio de la región Metropolitana (35,74\%), aunque la región Centro-Oeste mantiene un lugar importante (22,68\%). En el caso de revistas extranjeras, la concentración es mucho más fuerte, ya que la región Metropolitana reúne un $63,85 \%$ de los artículos, apareciendo luego la Bonaerense $(11,37)$ y la Centro-Oeste $(10,79)$. En los tres tipos de revistas es notable la baja participación de la región Nordeste. 
Revista de la Escuela de Ciencias de la Educación, año 10, número 9, enero a diciembre de 2014. Páginas 247-270. ISSN 1851-6297. ISSN EN LINEA 2362-3349. UN ACERCAMIENTO AL CAMPO ARGENTINO DE PRODUCCIÓN DE CONOCIMIENTO EDUCATIVO A TRAVÉS DE LAS PUBLICACIONES EN REVISTAS ACADÉMICAS DURANTE 2001-2010. JoRGe M. GoROSTIAGA Gimena Nieto - Florencia Cueli.

Mientras que la primacía de la región Metropolitana puede explicarse por la alta concentración de instituciones así como de los posgrados en educación (10) en esa región, llama la atención el alto porcentaje que presenta la región Centro-Oeste, sobre todo en el caso de las revistas argentinas, donde supera incluso al de la región Metropolitana. Aquí hay que tener en cuenta el ya mencionado peso dentro de nuestra base de los artículos de las revistas Alternativas Serie Espacio Pedagógico y de Cuadernos de Educación, editadas ambas por instituciones de la región Centro-Oeste. El peso de esas dos revistas influye en sesgar la distribución de las instituciones de pertenencia (dato a partir del cual se genera la distribución por regiones) por los altos porcentajes de publicación de autores de la propia institución que tienen ambas revistas (11). Otro dato a tener en cuenta es que para las categorías I, II y III del Programa de Incentivos de la SPU, que incluyen a los investigadores habilitados a dirigir proyectos, en el año 2000 la región Centro-Oeste era la que sumaba más investigadores en educación (102), seguida por la Noroeste, con 99, y la Metropolitana, con 81 (Galarza, 2007). Si por un lado este dato podría servir para explicar, al menos en parte, la alta productividad de la región Centro-Oeste, también llama la atención sobre el bajo porcentaje de artículos de parte de investigadores de la Región Noroeste en nuestra base (entre un $12 \%$ en las revistas de ciencias sociales y humanas y menos del $2 \%$ en las extranjeras).

\section{CUADRO 2}

Artículos seqún región a la que pertenecen las instituciones sede del primer autor. En porcentajes

\begin{tabular}{|l|c|c|c|c|}
\hline \multicolumn{1}{|c|}{ Región } & $\begin{array}{c}\text { Revistas } \\
\text { de educación }\end{array}$ & $\begin{array}{c}\text { Revistas de } \\
\text { ciencias sociales } \\
\text { y humanas }\end{array}$ & $\begin{array}{c}\text { Revistas } \\
\text { extranjeras }\end{array}$ & $\begin{array}{c}\text { Total de } \\
\text { revistas }\end{array}$ \\
\hline Metropolitana & 26,92 & 35,74 & 63,85 & 34,09 \\
\hline Centro-Oeste & 31,68 & 23,37 & 10,79 & 27,16 \\
\hline Centro-Este & 11,27 & 15,46 & 8,16 & 11,35 \\
\hline Bonaerense & 11,21 & 6,19 & 11,37 & 10,55 \\
\hline Sur & 11,88 & 5,50 & 2,62 & 9,51 \\
\hline Noroeste & 5,50 & 12,71 & 1,17 & 5,79 \\
\hline Nordeste & 1,54 & 1,03 & 2,04 & 1,55 \\
\hline \multicolumn{1}{|c|}{ TOTAL } & 100 & 100 & 100 & 100 \\
\hline
\end{tabular}

El análisis por sector institucional (véase Cuadro 3) muestra que las universidades públicas tienen un peso muy fuerte en los tres tipos de revistas: un 
Revista de la Escuela de Ciencias de la Educación, año 10, número 9, enero a diciembre de 2014. Páginas 247-270. ISSN 1851-6297. ISSN EN LINEA 2362-3349. UN ACERCAMIENTO AL CAMPO ARGENTINO DE PRODUCCIÓN DE CONOCIMIENTO

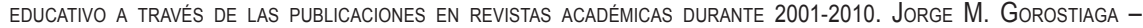
Gimena Nieto - Florencia Cuell.

$83,09 \%$ en las de educación, un $89,69 \%$ en las de ciencias sociales y humanas, y un $69,68 \%$ en las extranjeras. En el total de los tres conjuntos de artículos, las universidades privadas y los centros independientes, cada uno rondando entre un 5 y un $6 \%$, aparecen después de las universidades públicas. El aporte de los centros independientes cobra significancia en las revistas extranjeras $(12,54 \%)$, categoría en la que se destaca la cantidad de artículos firmados por investigadores de la Facultad Latinoamericana de Ciencias Sociales (FLACSO), con un total de 26 trabajos. Un elemento que las tres bases parecen reflejar es la debilidad del sector de universidades privadas en términos de investigación, a pesar de tener cuatro de los nueve doctorados en educación del país que estaban acreditados ante la Comisión Nacional de Evaluación y Acreditación Universitaria (CONEAU) en 2008 (De La Fare, 2008). Los institutos terciarios y secundarios tienen una presencia muy poco significativa, sobre todo en las revistas de ciencias sociales y humanas y en las extranjeras. Más pequeña aún es la participación de organismos internacionales y organismos gubernamentales, aunque este último sector alcanza más de un $5 \%$ en los artículos de revistas extranjeras.

\section{CUADRO 3}

Artículos según sector institucional al que pertenece el primer autor, en porcentajes

\begin{tabular}{|l|c|c|c|c|}
\hline Sector institucional & $\begin{array}{c}\text { Revistas } \\
\text { de educación }\end{array}$ & $\begin{array}{c}\text { Revistas de } \\
\text { ciencias sociales } \\
\text { y humanas }\end{array}$ & $\begin{array}{c}\text { Revistas } \\
\text { extranjeras }\end{array}$ & Total \\
\hline $\begin{array}{l}\text { Universidades } \\
\text { públicas }\end{array}$ & 85,25 & 89,69 & 69,68 & 83,35 \\
\hline $\begin{array}{l}\text { Universidades } \\
\text { privadas }\end{array}$ & 5,09 & 4,47 & 8,75 & 5,60 \\
\hline $\begin{array}{l}\text { Centros } \\
\text { independientes }\end{array}$ & 4,09 & 2,06 & 12,53 & 5,18 \\
\hline $\begin{array}{l}\text { Institutos terciarios } \\
\text { y secundarios }\end{array}$ & 3,35 & 1,03 & 1,17 & 2,68 \\
\hline $\begin{array}{l}\text { Organismos de } \\
\text { C y T }\end{array}$ & 1,41 & 2,40 & 0,29 & 1,36 \\
\hline $\begin{array}{l}\text { Organismos } \\
\text { gubernamentales }\end{array}$ & 0,54 & 0,35 & 5,83 & 1,36 \\
\hline $\begin{array}{l}\text { Organismos } \\
\text { internacionales }\end{array}$ & 0,27 & -- & 1,75 & 0,47 \\
\hline Total & 100 & 100 & 100 & 100 \\
\hline
\end{tabular}


Revista de la Escuela de Ciencias de la Educación, año 10, número 9, enero a diciembre de 2014. Páginas 247-270. ISSN 1851-6297. ISSN EN LINEA 2362-3349. UN ACERCAMIENTO AL CAMPO ARGENTINO DE PRODUCCIÓN DE CONOCIMIENTO EDUCATIVO A TRAVÉS DE LAS PUBLICACIONES EN REVISTAS ACADÉMICAS DURANTE 2001-2010. JoRGe M. GoROSTIAGA Gimena Nieto - Florencia Cueli.

El Cuadro 4, por su parte, muestra la distribución de artículos por institución dentro del sector universidades públicas (incluyendo en el cuadro sólo aquéllas que suman más de un $1 \%$ del total de artículos), distribución que revela un panorama de gran heterogeneidad. Para las revistas argentinas, se destacan, en primer término, la UBA $(18,38 \%$ ) y la UNSL $(14,46 \%)$, seguidas por la UNC $(11,16 \%)$. Puede notarse que las diez universidades que más artículos publican poseen revistas propias, las que, como señalamos antes, tienen cierto sesgo -variable según el caso- a favor de autores de la propia institución. En las revistas de ciencias sociales y humanas -en las cuales se repite, al menos para el caso de los artículos sobre educación, una fuerte endogamia institucional (12)- aumenta el predominio de artículos de la UBA $(29,88 \%)$, seguida nuevamente por la UNSL (16,86\%). Cabe notar que los artículos de autores de la UNER y la UNJu, con altos porcentajes en esta categoría, corresponden exclusivamente a revistas propias de cada una de estas dos universidades.

En el caso de los artículos publicados en revistas extranjeras, existe una concentración aún más importante en la UBA $(32,35 \%)$, mientras que otras universidades nacionales como las de Quilmes, Rosario, Córdoba y La Plata muestran un aporte muy significativo. La mayoría de las universidades de las regiones Nordeste, Noroeste y Sur no suman artículos en revistas extranjeras.

\section{CUADRO 4}

Artículos según universidades públicas, en porcentajes

\begin{tabular}{|l|c|c|c|c|}
\hline Universidad & $\begin{array}{c}\text { Revistas de } \\
\text { educación }\end{array}$ & $\begin{array}{c}\text { Revistas de } \\
\text { ciencias sociales } \\
\text { y humanas }\end{array}$ & $\begin{array}{c}\text { Revistas } \\
\text { extranjeras }\end{array}$ & Total \\
\hline UBA & 18,38 & 29,88 & 32,21 & 21,75 \\
\hline UNSL & 14,46 & 16,86 & 2,09 & 13,15 \\
\hline UNC & 11,16 & 4,98 & 7,11 & 9,70 \\
\hline UNCOMA & 8,57 & 5,36 & 1,67 & 7,17 \\
\hline UNR & 6,21 & 2,68 & 8,37 & 5,98 \\
\hline UNLP & 4,09 & 4,60 & 7,11 & 4,57 \\
\hline UNT & 4,95 & 1,92 & -- & 3,84 \\
\hline UNICEN & 4,09 & -- & 2,93 & 3,33 \\
\hline UNRC & 3,85 & 1,53 & 2,09 & 3,27 \\
\hline UNER & 1,49 & 12,26 & -- & 2,88 \\
\hline
\end{tabular}

(continúa en pág. 257) 
Revista de la Escuela de Ciencias de la Educación, año 10, número 9, enero a diciembre de 2014. Páginas 247-270. ISSN 1851-6297. ISSN EN LINEA 2362-3349. UN ACERCAMIENTO AL CAMPO ARGENTINO DE PRODUCCIÓN DE CONOCIMIENTO

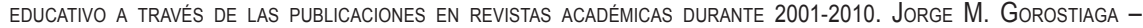
Gimena Nieto - Florencia Cuell.

(viene de pág. 256)

\begin{tabular}{|l|c|c|c|c|}
\hline Universidad & $\begin{array}{c}\text { Revistas de } \\
\text { educación }\end{array}$ & $\begin{array}{c}\text { Revistas de } \\
\text { ciencias sociales } \\
\text { y humanas }\end{array}$ & $\begin{array}{c}\text { Revistas } \\
\text { extranjeras }\end{array}$ & Total \\
\hline UNLPam & 3,30 & -- & 0,42 & 2,43 \\
\hline UNQ & 0,86 & 0,38 & 11,71 & 2,26 \\
\hline UNMdP & 2,20 & 1,50 & 3,35 & 2,20 \\
\hline UNL & 2,36 & 0,38 & 2,09 & 2,03 \\
\hline UNJu & 0,55 & 9,19 & 0,42 & 1,80 \\
\hline UNCuyo & 1,65 & 1,92 & 2,51 & 1,80 \\
\hline UNLu & 1,49 & 0,77 & 2,51 & 1,52 \\
\hline UNSAM & 0,94 & 0,38 & 3,76 & 1,24 \\
\hline UTN & 1,41 & 0,77 & 0,42 & 1,18 \\
\hline
\end{tabular}

En la comparación entre las tres categorías de revistas, llama la atención el abrupto descenso del porcentaje en las revistas extranjeras para la UNSL, y que instituciones con cierta presencia en revistas argentinas no están representadas en las revistas extranjeras, como es el caso de la UNER y la Universidad Nacional de Tucumán; o, a la inversa y más notoriamente dada la cantidad total de artículos en cada base, que una universidad (Universidad Nacional de Quilmes) presente más artículos en términos absolutos en las revistas extranjeras que en las nacionales. La dispar participación de las universidades nacionales tanto en revistas nacionales como extranjeras refleja, probablemente, la fuerte heterogeneidad existente dentro de este sector en términos de, entre otros aspectos, condiciones de trabajo, organización y características de la comunidad académica, participación en redes nacionales e internacionales, y destinatarios de la investigación sobre educación que se realiza (véase Kupervaser (2013) para un análisis de estos aspectos en dos universidades nacionales no metropolitanas).

En el caso de las universidades privadas, se nota una gran dispersión, siendo muy pocas las universidades que publican con regularidad en algún tipo de revista. El total de 119 artículos se distribuye entre 21 instituciones, pero sólo siete de ellas publicaron 3 o más artículos en total durante el período. También existe una gran heterogeneidad entre los tres tipos de revistas en lo que respecta a qué universidades publican en cada una. El Cuadro 5 muestra la distribución de artículos publicados en los tres tipos de revistas para esas siete universidades, destacándose en el total de artículos el dominio de la 
Revista de la Escuela de Ciencias de la Educación, año 10, número 9, enero a diciembre de 2014. Páginas 247-270. ISSN 1851-6297. ISSN EN LINEA 2362-3349. UN ACERCAMIENTO AL CAMPO ARGENTINO DE PRODUCCIÓN DE CONOCIMIENTO EDUCATIVO A TRAVÉS DE LAS PUBLICACIONES EN REVISTAS ACADÉMICAS DURANTE 2001-2010. JoRGe M. GoROSTIAGA Gimena Nieto - Florencia Cueli.

Universidad Católica de Córdoba (UCC) $(27,73 \%$ ) y de la Universidad de San Andrés (UDESA) (21,83\%). Ésta última se destaca por sumar más artículos en revistas extranjeras (14) que en las argentinas (12), situación que también se verifica para la Universidad Torcuato Di Tella (UTDT), aunque en otra magnitud (4 y 1 artículos, respectivamente).

CUADRO 5

Artículos según universidades privadas, en porcentajes

\begin{tabular}{|l|c|c|c|c|}
\hline Universidad & $\begin{array}{c}\text { Revistas de } \\
\text { educación }\end{array}$ & $\begin{array}{c}\text { Revistas de } \\
\text { ciencias sociales } \\
\text { y humanas }\end{array}$ & $\begin{array}{c}\text { Revistas } \\
\text { extranjeras }\end{array}$ & Total \\
\hline UCC & 42,10 & ---- & 3,33 & 27,73 \\
\hline UDESA & 15,79 & ---- & 46,66 & 21,85 \\
\hline USAL & 5,26 & 76,92 & ---- & 11,76 \\
\hline UCSF & 15,79 & ---- & ---- & 10,08 \\
\hline UCA & 7,89 & ---- & 10 & 7,56 \\
\hline UTDT & 1,31 & ---- & 13,33 & 4,20 \\
\hline CAECE & 2,63 & ---- & 3,33 & 2,52 \\
\hline $\begin{array}{l}\text { Univ. del } \\
\text { Aconcagua }\end{array}$ & 2,63 & 7,69 & ---- & 2,52 \\
\hline
\end{tabular}

Nuevamente, debe notarse una aparente endogamia de las revistas (en este caso de aquellas publicadas por universidades privadas) que podría estar sesgando los resultados. Así, entre las revistas de educación, los 12 artículos firmados por autores de la Universidad Católica de Santa Fe (UCSF) fueron publicados en la revista Krinein, editada por la misma universidad; de los 32 de la UCC, 27 fueron publicados en su revista Diálogos Pedagógicos. En el caso de las revistas de ciencias sociales y humanas, esta endogamia se manifiesta en la importante cantidad de artículos (diez) publicados por investigadores de la Universidad del Salvador (USAL) en su revista Psicología y Psicopedagogía.

Una forma posible de estimar la influencia de las distintas universidades sobre el campo de la producción, evitando el sesgo que genera la publicación de artículos elaborados por la propia institución, es la de considerar sólo los artículos que no cumplen esa condición. Esto modifica los porcentajes correspondientes a las revistas argentinas (de educación y de ciencias sociales y humanas) y, por lo tanto, los porcentajes de la mayoría de las universidades para el total de artículos, que ahora se reduce de 1772 a 1024. Así, pierden peso la UNC 
Revista de la Escuela de Ciencias de la Educación, año 10, número 9, enero a diciembre de 2014. Páginas 247-270. ISSN 1851-6297. ISSN EN LINEA 2362-3349. UN ACERCAMIENTO AL CAMPO ARGENTINO DE PRODUCCIÓN DE CONOCIMIENTO

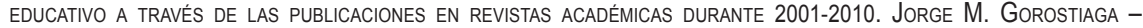
Gimena Nieto - Florencia Cuell.

$y$, sobre todo, la UNSL, mientras que la UBA cobra mayor predominio (véase Cuadro 6 , donde aparecen las universidades que suman más de un $2 \%$ del total). Exceptuando a esta última, aparecen tres grupos de universidades con valores cercanos, el primero constituido por la Universidad Nacional del Comahue (UNCOMA), la UNC, la UNLP y la Universidad Nacional de Rosario (UNR).

\section{CUADRO 6}

Artículos según universidades públicas, en porcentajes (sólo artículos publicados en revistas editadas por una institución distinta a la del 1er autor)

\begin{tabular}{|l|c|c|c|c|}
\hline Universidad & $\begin{array}{c}\text { Revistas de } \\
\text { educación }\end{array}$ & $\begin{array}{c}\text { Revistas de } \\
\text { ciencias sociales } \\
\text { y humanas }\end{array}$ & $\begin{array}{c}\text { Revistas } \\
\text { extranjeras }\end{array}$ & Total \\
\hline UBA & 24,96 & 26,19 & 32,21 & 26,76 \\
\hline UNCOMA & 8,42 & 5,95 & 1,67 & 6,64 \\
\hline UNC & 6,13 & 8,33 & 7,11 & 6,54 \\
\hline UNLP & 5,85 & 5,95 & 7,11 & 6,15 \\
\hline UNR & 4,99 & 8,33 & 8,37 & 6,05 \\
\hline UNQ & 1,57 & 1,19 & 11,71 & 3,91 \\
\hline UNMdP & 3,71 & 3,57 & 3,35 & 3,61 \\
\hline UNL & 4,28 & 1,19 & 2,09 & 3,51 \\
\hline UNRC & 3,85 & 4,76 & 2,09 & 3,51 \\
\hline UNICEN & 3,85 & -- & 2,93 & 3,32 \\
\hline UNCuyo & 2,99 & 2,38 & 2,51 & 2,83 \\
\hline UNLu & 2,71 & 2,38 & 2,51 & 2,64 \\
\hline UNT & 2,71 & 5,95 & --- & 2,34 \\
\hline UNSAM & 1,71 & 1,19 & 3,76 & 2,15 \\
\hline UNSL & 2,00 & 2,38 & 2,09 & 2,05 \\
\hline UTN & 2,57 & 2,38 & 0,42 & 2,05 \\
\hline
\end{tabular}

Aplicando el mismo criterio al conjunto de universidades privadas, también se observan cambios significativos. En este caso, el Cuadro 7 considera sólo las universidades que en total publicaron al menos 3 artículos, aunque los 
Revista de la Escuela de Ciencias de la Educación, año 10, número 9, enero a diciembre de 2014. Páginas 247-270. ISSN 1851-6297. ISSN EN LINEA 2362-3349. UN ACERCAMIENTO AL CAMPO ARGENTINO DE PRODUCCIÓN DE CONOCIMIENTO EDUCATIVO A TRAVÉS DE LAS PUBLICACIONES EN REVISTAS ACADÉMICAS DURANTE 2001-2010. JoRGe M. GoROSTIAGA Gimena Nieto - Florencia Cueli.

porcentajes, como en los casos anteriores, están calculados sobre el total de universidades. La base conjunta se ha reducido de 119 a 58 artículos. Llama la atención, por un lado, el aumento porcentual de la UDESA, colocándose en una posición de claro predominio sobre el resto. Por el otro lado, es notable la pérdida de peso de la UCC y la desaparición de la UCSF.

\section{CUADRO 7}

Artículos según universidades privadas, en porcentajes (sólo artículos publicados en revistas editadas por una institución distinta a la del 1er autor)

\begin{tabular}{|l|c|c|c|c|}
\hline Universidad & $\begin{array}{c}\text { Revistas } \\
\text { argentinas }\end{array}$ & $\begin{array}{c}\text { Revistas de } \\
\text { ciencias sociales } \\
\text { y humanas }\end{array}$ & $\begin{array}{c}\text { Revistas } \\
\text { extranjeras }\end{array}$ & Total \\
\hline UDESA & 37,50 & --- & 46,66 & 39,65 \\
\hline UCA & 25,00 & --- & 10 & 15,52 \\
\hline UCC & 20,83 & --- & 3,33 & 10,34 \\
\hline USAL & 16,66 & 25,00 & --- & 8,62 \\
\hline UTDT & 4,16 & --- & 13,33 & 8,62 \\
\hline CAECE & 8,33 & --- & 3,33 & 5,17 \\
\hline $\begin{array}{l}\text { Univ. del } \\
\text { Aconcagua }\end{array}$ & 8,33 & 25,00 & --- & 5,17 \\
\hline
\end{tabular}

El último aspecto a considerar en nuestro análisis es el de la distribución de la producción de acuerdo al área disciplinaria. Para el total de los artículos (véase Cuadro 8) hay un grupo de áreas que se sitúan entre alrededor de un 16 y un $11 \%$ (Sociología de la Educación, Didácticas Específicas, Psicología de la Educación y Política Educativa), seguidas por otro grupo que ronda entre el 6 y el 9\% (Historia de la Educación, Didáctica, Pedagogía y Formación Docente). El resto de las áreas presenta valores menores al 4\%, destacándose la baja presencia de Economía de la Educación, y de temáticas como la Educación Artística y la Educación de Adultos.

En las revistas argentinas de educación, el área de las Didácticas Específicas es la que concentra mayor porcentaje de artículos (15,92\%), seguida por Sociología de la Educación (13,01\%). Hay un grupo de áreas en el que cada una (Historia de la Educación, Didáctica, Política Educativa, Psicología de la Educación, Pedagogía y Formación Docente) suma entre un $10 \%$ y un $7 \%$ de los artículos, mientras que las áreas restantes se encuentran por debajo 
Revista de la Escuela de Ciencias de la Educación, año 10, número 9, enero a diciembre de 2014. Páginas 247-270. ISSN 1851-6297. ISSN EN LINEA 2362-3349. UN ACERCAMIENTO AL CAMPO ARGENTINO DE PRODUCCIÓN DE CONOCIMIENTO

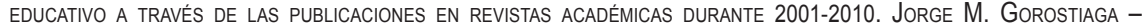
Gimena Nieto - Florencia Cuell.

del $5 \%$ cada una. En esta distribución puede estar influyendo el peso de las revistas más especializadas, que tienden a concentrarse en distintos campos de la didáctica, como enseñanza de las ciencias, física y matemática.

\section{CUADRO 8}

Artículos por área disciplinaria, en porcentajes

\begin{tabular}{|l|c|c|c|c|}
\hline Área disciplinaria & $\begin{array}{c}\text { Revistas de } \\
\text { educación }\end{array}$ & $\begin{array}{c}\text { Revistas de } \\
\text { ciencias sociales } \\
\text { y humanas }\end{array}$ & $\begin{array}{c}\text { Revistas } \\
\text { extranjeras }\end{array}$ & Totales \\
\hline $\begin{array}{l}\text { Sociología de la } \\
\text { Educación }\end{array}$ & 13,01 & 20,27 & 20,35 & 15,17 \\
\hline $\begin{array}{l}\text { Didácticas } \\
\text { Específicas }\end{array}$ & 15,92 & 6,87 & 9,01 & 13,59 \\
\hline $\begin{array}{l}\text { Psicología de la } \\
\text { Educación }\end{array}$ & 9,71 & 28,18 & 5,23 & 11,49 \\
\hline Política Educativa & 8,32 & 8,25 & 25,87 & 11,12 \\
\hline $\begin{array}{l}\text { Historia de la } \\
\text { Educación }\end{array}$ & 8,92 & 4,81 & 7,85 & 8,19 \\
\hline Didáctica & 9,84 & 4,12 & 4,07 & 8,14 \\
\hline Pedagogía & 7,59 & 4,47 & 4,36 & 6,65 \\
\hline $\begin{array}{l}\text { Formación } \\
\text { Docente }\end{array}$ & 7,40 & 1,37 & 5,23 & 6,24 \\
\hline $\begin{array}{l}\text { Tecnología } \\
\text { Educativa }\end{array}$ & 4,03 & 2,06 & 2,61 & 3,54 \\
\hline $\begin{array}{l}\text { Administración } \\
\text { del Sistema e } \\
\text { Instituciones } \\
\text { Educativas }\end{array}$ & 3,37 & 2,41 & 4,94 & 3,49 \\
\hline $\begin{array}{l}\text { Filosofía de la } \\
\text { Educación }\end{array}$ & 2,51 & 2,41 & 2,03 & 2,42 \\
\hline $\begin{array}{l}\text { Estudios del } \\
\text { Currículo }\end{array}$ & 2,51 & 2,75 & 1,45 & 2,37 \\
\hline $\begin{array}{l}\text { Antropología de la } \\
\text { Educación }\end{array}$ & 1,65 & 5,84 & 2,61 & 2,37 \\
\hline
\end{tabular}

(continúa en pág. 262) 
Revista de la Escuela de Ciencias de la Edducación, año 10, número 9, enero a diciembre de 2014. Páginas 247-270. ISSN 1851-6297. ISSN EN LINEA 2362-3349. UN ACERCAMIENTO AL CAMPO ARGENTINO DE PRODUCCIÓN DE CONOCIMIENTO EDUCATIVO A tRAVÉS DE LAS PUBLICACIONES EN REVISTAS ACADÉMICAS DURANTE 2001-2010. JoRge M. GoROSTIAGA Gimena Nieto - Florencia Cueli.

(viene de pág. 261)

\begin{tabular}{|l|c|c|c|c|}
\hline Área disciplinaria & $\begin{array}{c}\text { Revistas de } \\
\text { educación }\end{array}$ & $\begin{array}{c}\text { Revistas de } \\
\text { ciencias sociales } \\
\text { y humanas }\end{array}$ & $\begin{array}{c}\text { Revistas } \\
\text { extranjeras }\end{array}$ & Totales \\
\hline $\begin{array}{l}\text { Educación } \\
\text { Especial }\end{array}$ & 2,44 & 0,34 & --- & 1,77 \\
\hline $\begin{array}{l}\text { Educación } \\
\text { artística }\end{array}$ & 1,39 & 1,03 & 0,56 & 1,21 \\
\hline $\begin{array}{l}\text { Educación de } \\
\text { Adultos }\end{array}$ & 0,73 & 0,69 & 1,74 & 0,88 \\
\hline $\begin{array}{l}\text { Economía de la } \\
\text { Educación }\end{array}$ & 0,13 & 2,75 & 1,16 & 0,65 \\
\hline Otras & 0,53 & 1,37 & 0,87 & 0,70 \\
\hline Total & 100 & 100 & 100 & 100 \\
\hline
\end{tabular}

Dentro de las revistas de ciencias sociales y humanas es claro que en la distribución influye la fuerte orientación disciplinaria de varias de las revistas incluidas en la muestra. Se destacan las áreas de Psicología y Sociología de la Educación, con un $28,18 \%$ y un 20,27\%, respectivamente. A pesar de que siguen manteniéndose en cifras relativamente bajas, también es notable el crecimiento (en comparación con las revistas educativas) de las áreas de Antropología y Economía de la Educación. En este sentido, los investigadores de esta última área parecen inclinados a publicar en revistas de la disciplina madre antes que en revistas educativas (14).

En las revistas extranjeras, en cambio, aparece un dominio muy fuerte de los artículos sobre Política Educativa $(25,87 \%)$ y Sociología de la Educación $(20,35 \%)$, lo que podría indicar que los investigadores de estas áreas son los que tienen más inclinación o éxito en publicar en estas revistas, pero aquí habría que considerar si estas áreas no son las que se prestan más o son más demandadas a la hora de incluir artículos de autores extranjeros por parte de las revistas. No obstante, Didácticas Específicas conserva un porcentaje importante de artículos $(9,01 \%)$.

Para el conjunto de los artículos de revistas, la distribución de las áreas disciplinarias por sector institucional (Cuadro 9) revela que el de las universidades públicas es el único sector que participa en todas, aunque las universidades privadas y los centros independientes cubren una gran mayoría. La mayor producción de las universidades públicas se orienta a Sociología de la Educación, Didácticas Específicas y Psicología de la Educación. En las universidades privadas predominan Política Educativa, Psicología de la 
Revista de la Escuela de Ciencias de la Educación, año 10, número 9, enero a diciembre de 2014. Páginas 247-270. ISSN 1851-6297. ISSN EN LINEA 2362-3349. UN ACERCAMIENTO AL CAMPO ARGENTINO DE PRODUCCIÓN DE CONOCIMIENTO

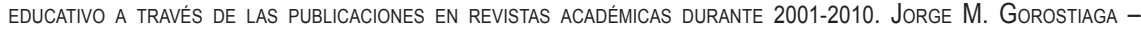
Gimena Nieto - Florencia Cueli.

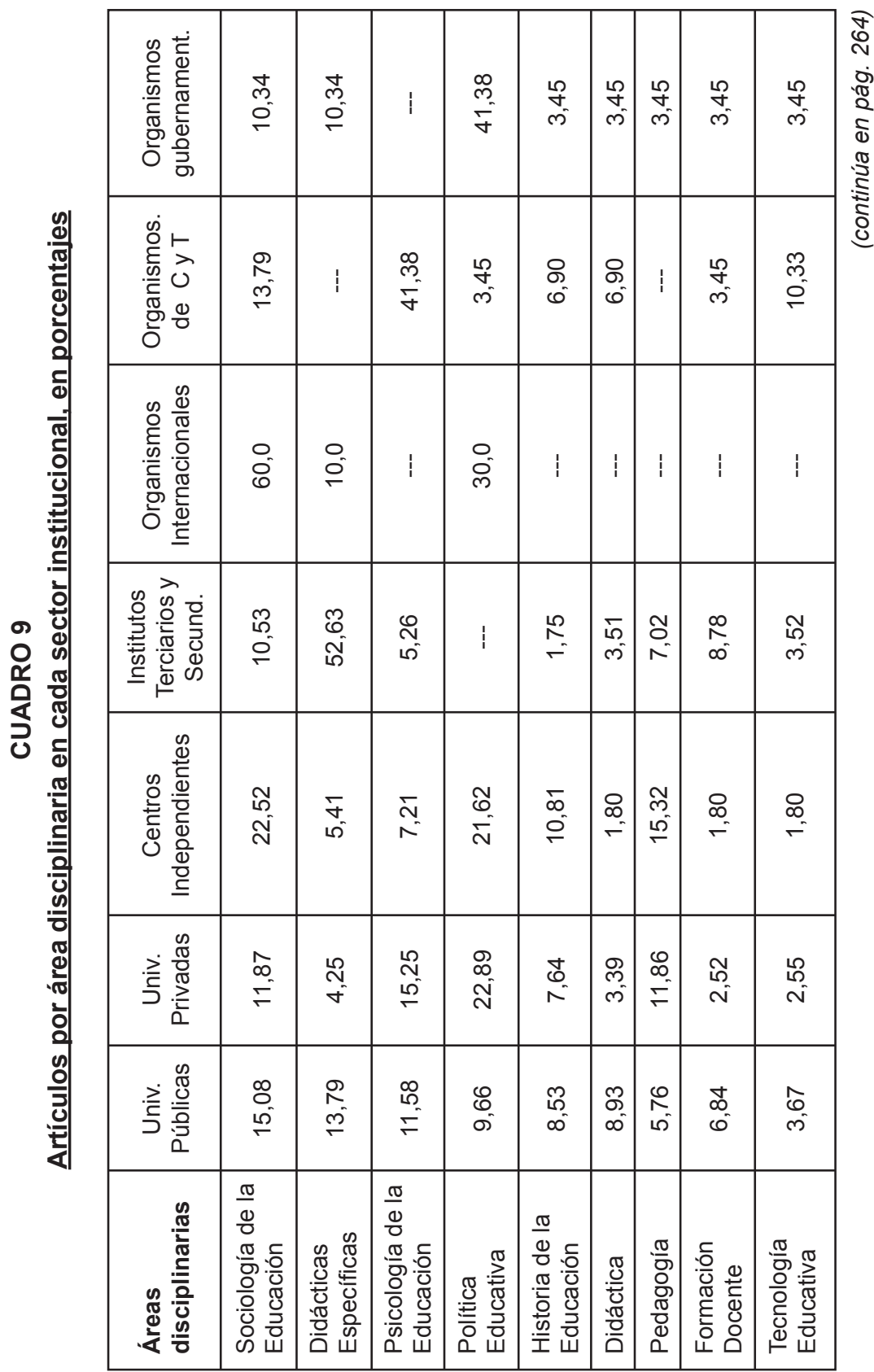


Revista de la Escuela de Ciencias de la Educación, año 10, número 9, enero a diciembre de 2014. Páginas 247-270. ISSN 1851-6297. ISSN EN LINEA 2362-3349. UN ACERCAMIENTO AL CAMPO ARGENTINO DE PRODUCCIÓN DE CONOCIMIENTO EDUCATIVO A tRAVÉS DE LAS PUBLICACIONES EN REVISTAS ACADÉmICAS DURANTE 2001-2010. JoRGE M. GoROStIAGA Gimena Nieto - Florencia Cueli.

\begin{tabular}{|c|c|c|c|c|c|c|c|c|c|c|}
\hline 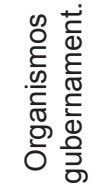 & $\begin{array}{l}\text { ले } \\
\text { ○. }\end{array}$ & $\dot{1}$ & $\begin{array}{l}\stackrel{f}{+} \\
\text { m) }\end{array}$ & $\mid$ & $\dot{1}$ & $\mid$ & $\begin{array}{l}8 \\
6\end{array}$ & $\mid$ & 1 & 으 \\
\hline 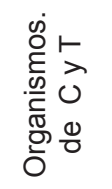 & $\begin{array}{l}\stackrel{5}{+} \\
\stackrel{5}{0}\end{array}$ & $\begin{array}{l}\stackrel{\text { fo }}{+} \\
\text { m. }\end{array}$ & $\mid$ & $\begin{array}{l}\stackrel{f}{+} \\
\text { m. }\end{array}$ & $\begin{array}{l}\stackrel{f}{+} \\
\text { m) }\end{array}$ & $\mid$ & i & $\mid$ & 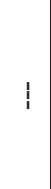 & 음 \\
\hline 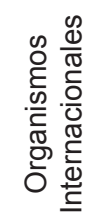 & i & 1 & 1 & 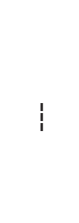 & 1 & $\mid$ & $\mid$ & 1 & $i$ & 음 \\
\hline 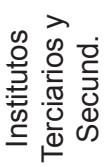 & 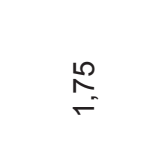 & $!$ & 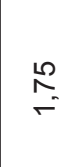 & $\mid$ & $\dot{1}$ & 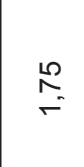 & $\stackrel{\stackrel{2}{r}}{r}$ & i & $\dot{1}$ & 으 \\
\hline 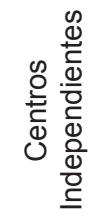 & $\frac{\text { P }}{\sim}$ & $\begin{array}{l}\text { 8 } \\
\text { ○ }\end{array}$ & $\begin{array}{l}\text { ¿ } \\
\text { m. }\end{array}$ & $\frac{R}{\sim}$ & $\mid$ & $\dot{1}$ & $\mid$ & $\stackrel{\infty}{-}$ & $\dot{1}$ & 8 \\
\hline 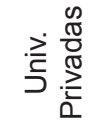 & $\begin{array}{l}\mathscr{8} \\
\text { ம் }\end{array}$ & $\begin{array}{l}\infty \\
0 \\
10\end{array}$ & $\stackrel{8}{6}$ & ஜ & $\begin{array}{l}\infty \\
\infty \\
0\end{array}$ & $\mid$ & $!$ & $\begin{array}{l}\infty \\
\infty \\
0\end{array}$ & $\underset{-}{8}$ & 은 \\
\hline ¿. & ले & 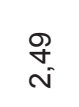 & $\stackrel{m}{\sim}$ & 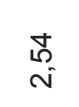 & $\stackrel{\text { న్ }}{\sigma}$ & $\stackrel{\sigma}{\check{\sigma}}$ & ᄋ̊ & $\begin{array}{l}\text { đै } \\
0 \\
0\end{array}$ & $\begin{array}{l}\infty \\
0 \\
0 \\
0\end{array}$ & 음 \\
\hline 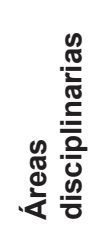 & 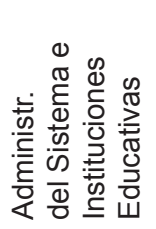 & 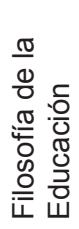 & 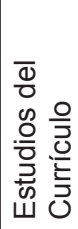 & 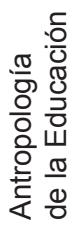 & 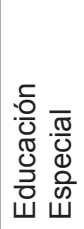 & 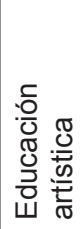 & 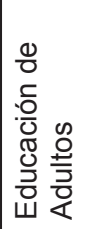 & 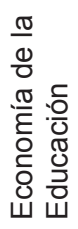 & 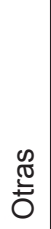 & $\frac{\pi}{0}$ \\
\hline
\end{tabular}


Revista de la Escuela de Ciencias de la Educación, año 10, número 9, enero a diciembre de 2014. Páginas 247-270. ISSN 1851-6297. ISSN EN LINEA 2362-3349. UN ACERCAMIENTO AL CAMPO ARGENTINO DE PRODUCCIÓN DE CONOCIMIENTO

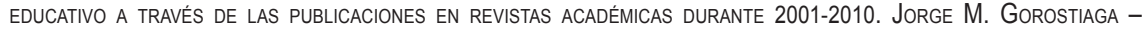
Gimena Nieto - Florencia Cuell.

Educación, Sociología de la Educación y Pedagogía, mientras que en los centros independientes, Sociología de la Educación, Política Educativa y Pedagogía. El sector más especializado es el de los organismos internacionales, que se concentra casi exclusivamente en Sociología de la Educación y Política Educativa. Los institutos terciarios y secundarios, por su parte, concentran más del $50 \%$ de sus artículos en el área de las Didácticas Específicas, mientras que organismos de ciencia y tecnología y organismos gubernamentales tienen una orientación muy fuerte hacia Psicología de la Educación y Política Educativa, respectivamente.

\section{Conclusiones}

La tendencia ascendente en la cantidad de artículos a lo largo de la década podría ser un signo de consolidación del campo académico. En este sentido, se destacan tanto el aumento de los artículos de revistas argentinas como la importante cantidad de artículos en nuestra base de revistas extranjeras (un promedio de 34 por año en una muestra de 42 revistas, muy superior al promedio de 17 por año correspondiente a la muestra de unas 60 revistas extranjeras del período 1997-2003 analizadas por Merodo et al. [2007] (15))

El análisis del volumen global de artículos de revistas revela que, si bien el grueso corresponde a revistas argentinas de educación, una parte significativa de la producción se canaliza hacia revistas de ciencias sociales y humanas, y hacia revistas de educación extranjeras. Una cuestión a explorar sería la de determinar cómo influyen en las tendencias de crecimiento y de distribución de la producción entre distintos tipos de revistas los incentivos, por parte de los organismos de ciencia y tecnología y de la SPU, que premian la publicación en revistas especializadas, especialmente las internacionales.

Los resultados de nuestro análisis dan cuenta también de que la publicación en revistas académicas tiende a concentrarse en la región Metropolitana y, en menor medida, en la Centro-Oeste, sumando entre las dos más del $60 \%$ de los artículos. La contracara es la baja producción de las regiones Noroeste y, particularmente, Nordeste. Esto no implica un juicio de valor acerca de la productividad -en términos de publicaciones- de los investigadores o de las instituciones de esas regiones, ya que pueden estar interviniendo otros factores como la distribución regional de los investigadores o el acceso desigual a recursos que facilitan la publicación de artículos.

En cuanto a los sectores institucionales, las universidades públicas lo hegemonizan fuertemente, hegemonía levemente atenuada en las revistas internacionales, en las que gana relevancia el sector de los centros independientes. Las universidades privadas, por su parte, muestran un nivel de producción muy pequeño en relación a su número y a su participación en el dictado de doctorados en educación. Por otra parte, se podría hipotetizar que sectores como el de los organismos internacionales, organismos gubernamentales e incluso 
Revista de la Escuela de Ciencias de la Educación, año 10, número 9, enero a diciembre de 2014. Páginas 247-270. ISSN 1851-6297. ISSN EN LINEA 2362-3349. UN ACERCAMIENTO AL CAMPO ARGENTINO DE PRODUCCIÓN DE CONOCIMIENTO

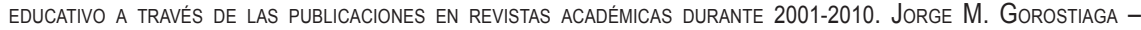
Gimena Nieto - Florencia Cueli.

algunas agencias del sector de centros independientes canalizan su producción prioritariamente hacia otros medios de difusión, como libros, informes o reportes.

Dentro de las universidades públicas, la UBA concentra el mayor porcentaje, seguida por un grupo de universidades de las regiones centrales del país (UNSL, UNC, UNR, UNLP) y la UNCOMA. A la vez, existe una fuerte heterogeneidad dentro del sector, ya que algunas universidades tienen una presencia similar en revistas argentinas y extranjeras, otras aparecen volcadas fuertemente hacia la producción en uno u otro tipo de revistas, y hay un grupo significativo, particularmente de las regiones Noroeste y Nordeste, con casi nula presencia en el volumen global de la producción de artículos.

No es posible determinar con los procedimientos utilizados cómo la existencia de revistas editadas por universidades sesga la base a favor de esas universidades; es decir si (o en qué medida) las publicaciones en esas revistas de artículos firmados por académicos de la propia institución reflejan directamente el grado de productividad de esas universidades, o si el nivel de publicaciones resulta inflado artificialmente. Si ocurriera esto último, estaría distorsionando nuestros resultados respecto a la distribución por regiones, por sector institucional y, dentro de éstos, por universidades públicas y privadas. Para estos dos últimos sectores mostramos cómo la utilización de una base diferente -incluyendo sólo los artículos publicados en revistas editadas por una institución distinta a la del primer autor de cada artículo- modifica sustancialmente los porcentajes de algunas universidades y aumenta el peso de las instituciones de las regiones metropolitana y bonaerense, pero no altera los rasgos más fundamentales como el predominio de la UBA dentro de las universidades públicas o el de las regiones centrales (Bonaerense, Centro Este, Centro Oeste y Metropolitana) para ambos tipos de universidades.

En la distribución por áreas disciplinares, se verifica una distribución heterogénea con algunas áreas dominantes (Sociología de la Educación, Didácticas Específicas, Psicología de la Educación y Política Educativa) y otras de muy escasa presencia (Economía de la Educación, Antropología de la Educación, Estudios del Currículo, Educación Artística, Especial y de Adultos). Las áreas con mayor presencia varían en las distintas bases de revistas: Didácticas Específicas en la de educación argentinas; Psicología de la Educación en la de ciencias sociales y humanas; y Política Educativa y Sociología de la Educación en la de extranjeras.

Otro aspecto a destacar es la continuidad con las principales tendencias identificadas para el período 1997-2003 por Merodo et al. (2007), sobre todo en cuanto a la concentración geográfica e institucional. Una diferencia significativa es el crecimiento de la región Sur, que se explicaría, en parte, por la inclusión en nuestra base de tres revistas editadas en dicha región que no eran parte de la muestra del estudio anterior. Respecto a las áreas disciplinarias, la diferencia más notoria es el retroceso general de Historia de la Educación, que 
Revista de la Escuela de Ciencias de la Educación, año 10, número 9, enero a diciembre de 2014. Páginas 247-270. ISSN 1851-6297. ISSN EN LINEA 2362-3349. UN ACERCAMIENTO AL CAMPO ARGENTINO DE PRODUCCIÓN DE CONOCIMIENTO

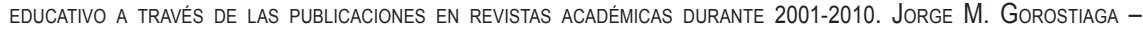
Gimena Nieto - Florencia Cueli.

antes aparecía como la de mayor porcentaje en artículos de revistas argentinas (en una muestra de 11 revistas, significativamente menor a la de este estudio).

En términos generales, algunos indicadores apuntan a cierta consolidación del campo, aunque persisten algunos rasgos que dan cuenta de sus debilidades (Palamidessi et al., 2012), como son los fuertes desbalances en términos de la distribución regional (en particular, la escasa producción de las regiones Noroeste y Nordeste tanto en revistas nacionales como extranjeras), por sector institucional (donde se destaca la hegemonía de las universidades públicas y la débil participación de las universidades privadas) y en términos del desarrollo de subdisciplinas (e.g., la baja presencia de Economía de la Educación). Estos resultados, obtenidos a través del análisis de los artículos publicados en revistas académicas, podrían complementarse o contrastarse con el análisis de otros medios de difusión (libros, ponencias, informes, etc.) para obtener un panorama más amplio de los rasgos del campo de la producción de conocimiento educativo en la Argentina.

\section{Notas Bibliográficas}

(1) Artículo realizado en base a resultados parciales del PICTO ANPCyT-UNSAM 2008-00043 "La producción y difusión de conocimiento en educación y las políticas educativas en Argentina (2001-2010)". Investigador Responsable: Jorge M. Gorostiaga.

(2) Colaboraron en la elaboración de las bases de datos: Graciela Caironi, Mariana Funes, Silvia Giuliano, María José Morard y María Elena Parodi

(3) Las revistas argentinas de educación relevadas son: Actas Pedagógicas (Univ. Nacional del Comahue); Alternativas. Serie: Espacios Pedagógicos (Univ. Nacional de San Luis); Alternativas. Serie: Historia y prácticas pedagógicas (Univ. Nacional de San Luis); Anuario de Historia de la Educación (Sociedad Argentina de Historia de la Educación); Archivos de Ciencias de la Educación (Univ. Nacional de La Plata); Boletín de Antropología y Educación (Univ. de Buenos Aires); Contextos de Educación (Univ. Nacional de Río Cuarto); Cuaderno de Pedagogía (Centro de Estudios en Pedagogía Crítica); Cuadernos de Educación (Univ. Nacional de Córdoba); Diálogos Pedagógicos (Univ. Católica de Córdoba); Educación, Lenguaje y Sociedad (Univ. Nacional de La Pampa); Espacios en Blanco (UNICEN); Krinein (Univ. Católica de Santa Fe); Páginas (Univ. Nacional de Córdoba); Pensamiento Universitario; Pilquen Sección Psicopedagogía (Univ. Nacional del Comahue); Praxis Educativa (Univ. Nacional de La Pampa); Premisa (Sociedad Argentina de Educación Matemática); Propuesta Educativa (Facultad Latinoamericana de Ciencias Sociales sede Argentina); Revista Argentina de Educación Superior (Univ. Nacional UNTREF); Revista Argentina de Educación (Asoc. Graduados en Ciencias de la Educación); Revista de Educación (Univ. Nacional de Mar del Plata); Revista de Enseñanza de la Física (Asoc. Profesores de Física); Revista de la Escuela de Ciencias de la Educación (Univ. Nacional de Rosario); Revista del Departamento de Ciencias de la Educación (Univ. Nacional de Tucumán); Revista de Política Educativa (Univ. de San Andrés); Revista del IICE (Univ. de Buenos Aires); Revista del IRICE (Instituto Rosario de Investigaciones en Ciencias de la Educación); Revista Electrónica de Investigación en Educación en Ciencias (Univ. Nacional del Centro de la Provincia de Buenos Aires); 
Revista de la Escuela de Ciencias de la Edducación, año 10, número 9, enero a diciembre de 2014. Páginas 247-270. ISSN 1851-6297. ISSN EN LÍNEA 2362-3349. UN ACERCAMIENTO AL CAMPO ARGENTINO DE PRODUCCIÓN DE CONOCIMIENTO EDUCATIVO A tRAVÉS DE LAS PUBLICACIONES EN REVISTAS ACAdÉmICAS DURANTE 2001-2010. JoRge M. GoROSTIAGA Gimena Nieto - Florencia Cueli.

Revista Iberoamericana de Tecnología en Educación y Educación en Tecnología (Red de Universidades Nacionales con carreras de Informática de Argentina).

(4) Como señala Kreimer (2011), a diferencia de las ciencias exactas y naturales, las normas para la evaluación y aprobación o rechazo de artículos en las revistas de ciencias sociales están lejos de ser homogéneas, ya que algunas tienen un comité colectivo que discute sobre los textos, otras tienen mecanismos de referato anónimo, en algunos casos es el editor quien toma las decisiones, y en otros ni siquiera hacen explícitos los procedimientos que emplean.

(5) Las revistas argentinas de ciencias sociales y humanidades relevadas son: Anuario de Filosofía Argentina y Americana (Univ. Nacional de Cuyo); Anuario de Investigaciones (Univ. de Buenos Aires); Ciencia, Docencia y Tecnología (Univ. Nacional de Entre Ríos), Cuadernos de Antropología Social (Univ. de Buenos Aires); Cuadernos de la Facultad de Humanidades y Ciencias Sociales (Univ. Nacional de Jujuy); Fundamentos en Humanidades (Univ. Nacional de San Luis); Interdisciplinaria (Centro Interamericano de Investigaciones Psicológicas y Ciencias Afines): Pilquen Ciencias Sociales (Univ. Nacional del Comahue): Psicología y Psicopedagogía (Univ. del Salvador); Revista Argentina de Sociología (Consejo de Profesionales en Sociología); Revista de Economía y Estadística (Univ. Nacional de Córdoba): y Revista de Psicología (Univ. Nacional de La Plata).

(6) Las revistas extranjeras relevadas son:

-Brasil: Avaliação: Revista da Avaliação da Educação Superior; Cadernos de Pesquisa; Educação e Pesquisa; Educaçao e Realidade; Educaçao e Sociedad; Educaçao em Revista; Educar em Revista; Historia da Educaçao; Pró-Posições; Revista Brasileira da Historia da Educaçao; y Revista Brasileira de Educação.

-España: Didáctica de las Ciencias Experimentales y Sociales; Enseñanza de las Ciencias; Historia de la Educación; Infancia y Aprendizaje; Revista Complutense de Educación; Revista de Educación; Revista de Psicodidáctica; Revista del Profesorado; Revista Electrónica de Investigación y Evaluación Educativa; Revista Electrónica Iberoamericana sobre Calidad, Eficacia y Cambio en Educación; Revista Española de Educación Comparada; y Revista Interuniversitaria.

-Estados Unidos: American Educational Research Journal; Anthropology \& Education Quarterly; Archivos Analíticos de Política Educativa; Comparative Education Review; e International Higher Education.

-Gran Bretaña. British Educational Research Journal; Comparative Education; Compare; Educational Psychology: An International Journal of Experimental Educational Psychology; Journal of Curriculum Studies; Journal of Education Policy; y Paedagogica Historica. -México: Perfiles Educativos; Revista de la Educación Superior; Revista Electrónica de Investigación Educativa; Revista Latinoamericana de Estudios Educativos; y Revista Mexicana de Investigación Educativa.

-organismos internacionales: Educación Superior y Sociedad (UNESCO); International Review of Education (UNESCO); y Revista Iberoamericana de Educación (OEI).

(7) La distribución de las provincias por región es la siguiente: Bonaerense: Provincia de Buenos Aires; Centro-Este: Entre Ríos y Santa Fe; Centro-Oeste: Córdoba, La Rioja, Mendoza, San Luis y San Juan; Metropolitana: Ciudad de Buenos Aires y Provincia de Buenos Aires; Nordeste: Chaco, Corrientes, Formosa y Misiones; Noroeste: Catamarca, Jujuy, Salta, Santiago del Estero y Tucumán; y Sur: Chubut, La Pampa, Neuquén, Río Negro, Santa Cruz y Tierra del Fuego.

En esta división, la Universidad Nacional de Lujan (UNLu) y la UNLP aparecen en dos regiones: la Metropolitana y la Bonaerense. Para este estudio se incluyeron ambas universidades en la región Bonaerense. 
Revista de la Escuela de Ciencias de la Educación, año 10, número 9, enero a diciembre de 2014. Páginas 247-270. ISSN 1851-6297. ISSN EN LINEA 2362-3349. UN ACERCAMIENTO AL CAMPO ARGENTINO DE PRODUCCIÓN DE CONOCIMIENTO

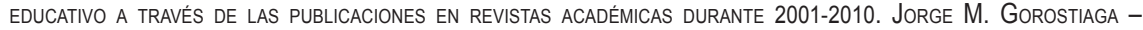
Gimena Nieto - Florencia Cueli.

(8) La gran mayoría de los investigadores del CONICET del área de ciencias sociales y humanas tiene sede de trabajo en universidades públicas o privadas.

(9) Estas categorías combinan áreas disciplinares con áreas temáticas, pero hemos optado por denominarlas áreas disciplinarias, siguiendo la terminología usada en Merodo et al. (2007). Con respecto a dicha clasificación sólo se introdujeron dos variantes: se eliminó la categoría "Reformas Educativas" por considerársela subsumible en "Política Educativa", y se agregó "Antropología de la Educación".

(10) De la Fare (2008) reporta que las regiones Metropolitana y Bonaerense juntas poseían el $47 \%$ de las carreras de posgrado en educación acreditadas ante CONEAU, mientras que un $20 \%$ pertenecía a la Centro-Oeste y un $11 \%$ a la Centro-Este (datos correspondientes al año 2008).

(11) Este sesgo a favor de los autores de la propia institución -endogamia considerada frecuente en las revistas de ciencias sociales (Kreimer, 2011)- es común en nuestra muestra a casi todas las publicaciones de universidades, tanto públicas como privadas, pero varía entre un $98 \%$ de los artículos (Revista del Departamento de Ciencias de la Educación, Universidad Nacional de Tucumán) a un 11\% (Revista Argentina de Educación Superior). Cuadernos de Educación y Alternativas Serie Espacio Pedagógico se ubican entre las de más alto porcentaje, con $69 \%$ y $60 \%$ de sus artículos firmados por autores de la propia institución, respectivamente. Nótese que estos porcentajes se calculan sin considerar los artículos publicados sólo por autores de instituciones extranjeras, cuya cantidad e incidencia dentro del total de artículos es también variable de acuerdo con las revistas.

(12) Es notable el caso del Anuario de Investigaciones de la Facultad de Psicología de la UBA, ya que publica sólo artículos producidos por investigadores de la propia facultad.

(13) Debe admitirse que aquí se introduce un sesgo contrario, en detrimento de las universidades que publican revistas, ya que los artículos publicados por académicos de la propia institución podrían haber sido eventualmente publicados en una revista editada por otra institución.

(14) En números absolutos, los artículos sobre Economía de la Educación suman dos en la base de revistas de educación y ocho en la de revistas de ciencias sociales y humanas, mientras que totalizan cuatro en la base de revistas educativas extranjeras.

(15) Esta comparación entre las dos muestras debe ser matizada por el hecho de que nuestra muestra abarca más años y estuvo levemente sesgada hacia revistas con antecedentes de publicación de autores de instituciones argentinas.

\section{Referencias Bibliográficas}

- $\quad$ Alvarenga, L. (2000). Contribuições para os estudos sobre a pesquisa educacional no Brasil: análise bibliométrica de artigos da Revista Brasileira de Estudos Pedagógicos (1944-1974). Revista Brasileira de Estudos Pedagógicos, 81 (198), 244-272.

- Anta, C. (2008). Análisis bibliométrico de la investigación educativa divulgada en publicaciones periódicas españolas entre 1990-2002. Revista Electrónica de Investigación Educativa, 10 (1). Recuperado de: http://redie.uabc.mx/vol10no1/contenido-anta.html

- De La Fare, M. (2008). La expansión de carreras de posgrado en Educación en Argentina. Archivos de Ciencias de la Educación, 2 (2), 103-120.

- Galán, M.I. y Rojas, C.L. (1995). Análisis de la producción en educativa. Las revistas mexicanas sobre educación en la década de los ochenta. México: UNAM-Centro de Investigaciones y Servicios Educativos. 
Revista de la Escuela de Ciencias de la Edducación, año 10, número 9, enero a diciembre de 2014. Páginas 247-270. ISSN 1851-6297. ISSN EN LÍNEA 2362-3349. UN ACERCAMIENTO AL CAMPO ARGENTINO DE PRODUCCIÓN DE CONOCIMIENTO EDUCATIVO A tRAVÉS DE LAS PUBLICACIONES EN REVISTAS ACAdÉmICAS DURANTE 2001-2010. JoRge M. GoROSTIAGA Gimena Nieto - Florencia Cueli.

- Galarza, D. (2007). Las universidades públicas. En M. Palamidessi, C. Suasnábar y D. Galarza (Eds.). Educación, conocimiento y política: Argentina 1983-2003 (pp. 99-122). Buenos Aires: FLACSO/Manantial.

- Kreimer, P. (2011). La evaluación de la actividad científica: desde la indagación sociológica a la burocratización. Dilemas actuales. Propuesta Educativa, 36 (2), 59-77.

- Kupervaser, A. S. (2013). La producción académica en educación: el caso de dos universidades públicas no metropolitanas de Argentina. En N. G. Gutiérrez Serrano (Ed.). Formación, política e investigación. Espacios de producción de conocimiento en educación en México y el Cono Sur, Tomo 2 (pp. 179-208). México: UNAM.

- Marquina, M. (2012). La profesión académica en Argentina: principales características a partir de las políticas recientes. En N. Fernández Lamarra y M. Marquina (Eds.). El futuro de la profesión académica: Desafíos para los países emergentes (pp. 126-147). Sáenz Peña: EDUNTREF.

- Merodo, A., Atairo, D., Stagno, L. y Palamidessi, M. (2007). La producción académica sobre educación en la Argentina (1997-2003). En M. Palamidessi, C. Suasnábar y D. Galarza (Eds.). Educación, conocimiento y política: Argentina 1983-2003 (pp. 187-207). Buenos Aires: FLACSO/Manantial.

- Palamidessi, M., Galarza, D. y Cardini, A. (2012). Un largo y sinuoso camino: las relaciones entre la política educativa y la producción de conocimientos sobre educación en Argentina. En M. Palamidessi, C. Suasnabar,y J. Gorostiaga (Eds.). Investigación educativa y política en América Latina (113-140). Buenos Aires: Noveduc.

- $\quad$ Palamidessi, M., Gorostiaga, J. y Suasnábar, C. (2014). El desarrollo de la investigación educativa y sus vinculaciones con el gobierno de la educación en América Latina. Perfiles Educativos, XXXVI (143), 49-66.

- $\quad$ Palamidessi, M. y Devetac, R. (2007). Las revistas académicas del campo de la educación (Argentina, 1990-2002). Archivos de Ciencias de la Educación, 1 (1), 131-158.

- $\quad$ Schriewer, J. \& Keiner, E. (1997). Pautas de comunicación y tradiciones intelectuales en las ciencias de la educación: Francia y Alemania. Revista Mexicana de Investigación Educativa, 2 (3), 117-148.

- Tarrés, M. C. (2009). Análisis bibliométrico de la Revista Medicina y Cine (2005-2008). Revista Medicina y Cine, 5 (3). Recuperado de: http://campus.usal.es/ revistamedicinacine/ Vol_5/5.3/esp.5.3.htlm/bibliom.htm

- Waldegg, G. (1997). La literatura científica. Revista Mexicana de Investigación Educativa, 2 (3), 149-156. 